\title{
Using polarization maintaining fibers for the purpose of a polarization multiplex
}

M. Kyselák, P. Šimák, F. Dvořák, Č. Vlček

M. Kyselák, P. Šimák, F. Dvořák, Č. Vlček, "Using polarization maintaining fibers for the purpose of a polarization multiplex," Proc. SPIE 10452, 14th Conference on Education and Training in Optics and Photonics: ETOP 2017, 104520G (16 August 2017); doi: 10.1117/12.2264692

SDIE Event: 14th Conference on Education and Training in Optics and Photonics, ETOP 2017, 2017, Hangzhou, China 


\title{
Using Polarization Maintaining Fibers for the Purpose of a Polarization Multiplex
}

\author{
M. Kyselák*a, P. Šimák ${ }^{\mathrm{b}}$, F. Dvořák ${ }^{\mathrm{c}}, \check{C ̆}^{\text {. }}$ Vlček $^{\mathrm{a}}$ \\ ${ }^{a}$ Department of Electrical Engineering, Faculty of Military Technology, University of Defense \\ Kounicova 65, Brno, Czech Republic; ${ }^{b}$ Department of Telecommunications, Brno University of \\ Technology, Technická 2, Czech Republic; ' Department of Radar Technology Faculty of Military \\ Technology, University of Defense Kounicova 65, Brno, Czech Republic
}

\begin{abstract}
Due to increasing demands for bandwidth are nowadays very popular optical networks. Installation of new fiber-optic networks is expensive, so is necessary to use existing fiber optic network very effectively. The main parameters that limit the optical networks are dispersion and attenuation. Efficiency optical networks could improve the use of two polarization axes, similar to the technology used in radio technologies. Use of fiber preserves polarization allows the use of two polarization planes. This article deals with the optical wavelength division multiplex in the polarization maintaining fibers.
\end{abstract}

Keywords: polarization, Stokes vectors, Jones vectors, Poincaré sphere, polarization multiplex

\section{INTRODUCTION}

If the light is not polarized, electric intensity vector $\mathrm{E}$ oscillates in a random direction perpendicular to the propagation direction. If propagates in a constant direction, it is a polarized light. The electric field $\mathrm{E}$ can be written to the $\mathrm{x}$-axis and $\mathrm{y}$-axis [1]:

$$
\begin{aligned}
& E_{x}(z, t)=E_{0 x} \cos \left(\omega t-k z+\delta_{x}\right), \\
& E_{y}(z, t)=E_{0 x} \cos \left(\omega t-k z+\delta_{y}\right),
\end{aligned}
$$

where $\omega=2 \pi f$, which is the angular frequency of the wave and $\mathrm{k}=2 \pi / \mu$ is the wave number, $\mathrm{E}_{0 \mathrm{x}}$ a $\mathrm{E}_{0 \mathrm{y}}$ are amplitudes and $\delta_{\mathrm{x}}, \delta_{\mathrm{y}}$ are phases. The expression $\omega t-k z$ is called propagator [1] and [3].

Eliminating the phase-spatial propagator $\omega t-k z$ from equations (1) and (2) we obtain the equation of an ellipse

$$
\frac{E_{x}(z, t)^{2}}{E_{0 x}^{2}}+\frac{E_{y}(z, t)^{2}}{E_{0 y}^{2}}-\frac{2 E_{x}(z, t)^{2} E_{y}(z, t)^{2}}{E_{0 x} E_{0 y}} \cos \delta=\sin ^{2} \delta,
$$

where $\delta=\delta_{y}-\delta_{x}$.

The equation is analytical expression of the ellipse. Polarization ellipse is described by two angular parameters and orientation angle

$$
\tan 2 \psi=\frac{2 E_{0 x} E_{0 y}}{E_{0 x}^{2}-E_{0 y}^{2}} \cos \delta, \quad 0 \leq \psi \leq \pi
$$

and elliptical angle

$$
\tan 2 \chi=\frac{2 E_{0 x} E_{0 y} \sin \delta}{E_{0 x}^{2}+E_{0 y}^{2}} \cos \delta, \quad \frac{-\pi}{4} \leq \chi \leq \frac{\pi}{4} .
$$

14th Conference on Education and Training in Optics and Photonics: ETOP 2017, edited by Xu Liu,

Xi-Cheng Zhang, Proc. of SPIE Vol. 10452, 104520G · C 2017 ICO, IEEE, OSA, SPIE

CCC code: $0277-786 \mathrm{X} / 17 / \$ 18 \cdot$ doi: $10.1117 / 12.2264692$

Proc. of SPIE Vol. 10452 104520G-1 
Using the Stokes parameters and Poincare sphere can identify the type and degree of polarization. Among the Stokes parameters $S_{0}$ to $S_{3}$ is valid [1]

$$
S_{0}^{2}=S_{1}^{2}+S_{2}^{2}+S_{3}^{2}
$$

where the parameters are defined:

$$
\begin{aligned}
& S_{0}=E_{0 x}^{2}+E_{0 y}^{2}, \\
& S_{1}=E_{0 x}^{2}-E_{0 y}^{2}, \\
& S_{2}=2 E_{0 x}^{2} E_{0 y}^{2} \cos \delta, \\
& S_{3}=2 E_{0 x}^{2} E_{0 y}^{2} \sin \delta, \quad \text { where } \delta=\delta_{y}-\delta_{x} .
\end{aligned}
$$

$\mathrm{S}_{0}$ parameter describes the intensity of light, the second parameter $\mathrm{S}_{1}$ describes the superiority of horizontal polarized light over vertical polarized light. The third parameter $S_{2}$ describes the superiority of $+45^{\circ}$ polarized lights above the $-45^{\circ}$ light and the fourth parameter $S_{3}$ describes the superiority of circular polarized light over linear polarized light. Practice is to write the Stokes parameters in a matrix, which is called the Stokes vector for elliptically polarized light

$$
\left[\begin{array}{l}
S_{0} \\
S_{1} \\
S_{2} \\
S_{3}
\end{array}\right]=\left[\begin{array}{c}
E_{0 x}^{2}+E_{0 y}^{2} \\
E_{0 x}^{2}-E_{0 y}^{2} \\
2 E_{0 x}^{2} E_{0 y}^{2} \cos \delta \\
2 E_{0 x}^{2} E_{0 y}^{2} \sin \delta
\end{array}\right] .
$$

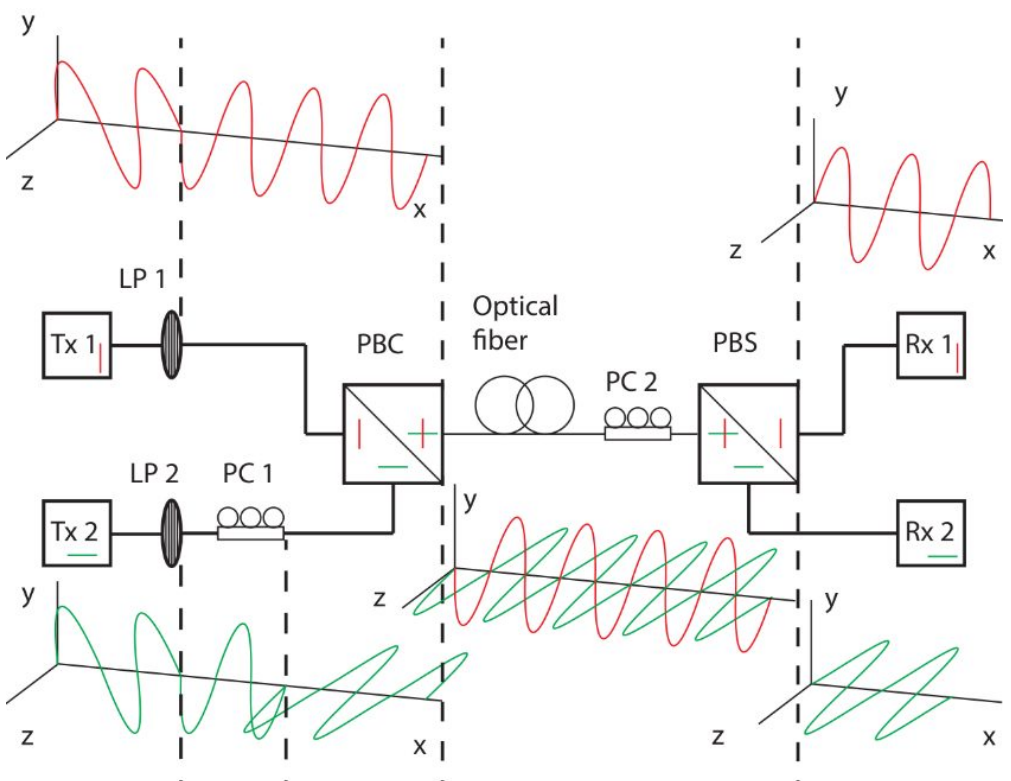

Fig. 1: The general arrangement of the polarization multiplexing, where Tx - Transmitter, Rx - Receiver, LP - Linear Polarizer, PC - Polarization Controller, PBC - Polarization Beam Coupler, PBS - Polarization Beam Splitter

\section{USE OF THE TWO POLARIZATION PLANES}

As mentioned, the optical signal propagates in the fiber in two polarization planes which are perpendicular to each other. The basic idea is to use polarization multiplexing each plane as an independent optical signal to transmit information. This doubles the bandwidth as both optical signals can have the same wavelength. 


\subsection{Basic consideration}

To realize the polarization multiplex they are required two signal sources, one with horizontal polarization and the other with vertical. Consequently, it is necessary to combine both signals into one conjoint fiber through the polarization beam combiner. To detect this multiplexed signals is necessary to keep the original polarization axes and both rotated at the correct angle. Polarization beam splitter arranges the correct rotation signals. Polarization beam splitter splits both signals and then simply have to bring signals to the right receiver. In figure 1 we can see the signals changing from both transmitters when passing through the various components. Axis up shows the signal from the transmitter Tx 1 and the axis below shows the involvement of a signal from the Tx 2 .

\subsection{Description of experimental workplace}

By the polarization splitter we can combine both optical signals into the polarization maintaining optical fiber. Both input signals are linearly polarized, because the SFP module has a light source laser diode. Therefore, we don't need to add linear fiber polarizers. While both input signals are linearly polarized, but have a bad angle of rotation, so both signals must be rotated to position linearly horizontal polarization using polarization controllers. Polarimeter observes the current state of polarization. The inputs of the polarization splitters are two signals with horizontal polarization, as can be seen in Figure 2.

One of the signal is rotated to the vertical polarization. Subsequently, the multiplexed signal passes through the polarization maintaining fiber, where the two axes vectorially summed together to form the right-handed polarized light, as shown on Fig. 3b. Thanks to the polarization maintaining fiber, the input of polarization beam splitter has same polarization as at the output of the polarization beam combiner. Second polarization beam splitter thus signal demultiplexes and then the two optical signals are inputted to the right receivers.

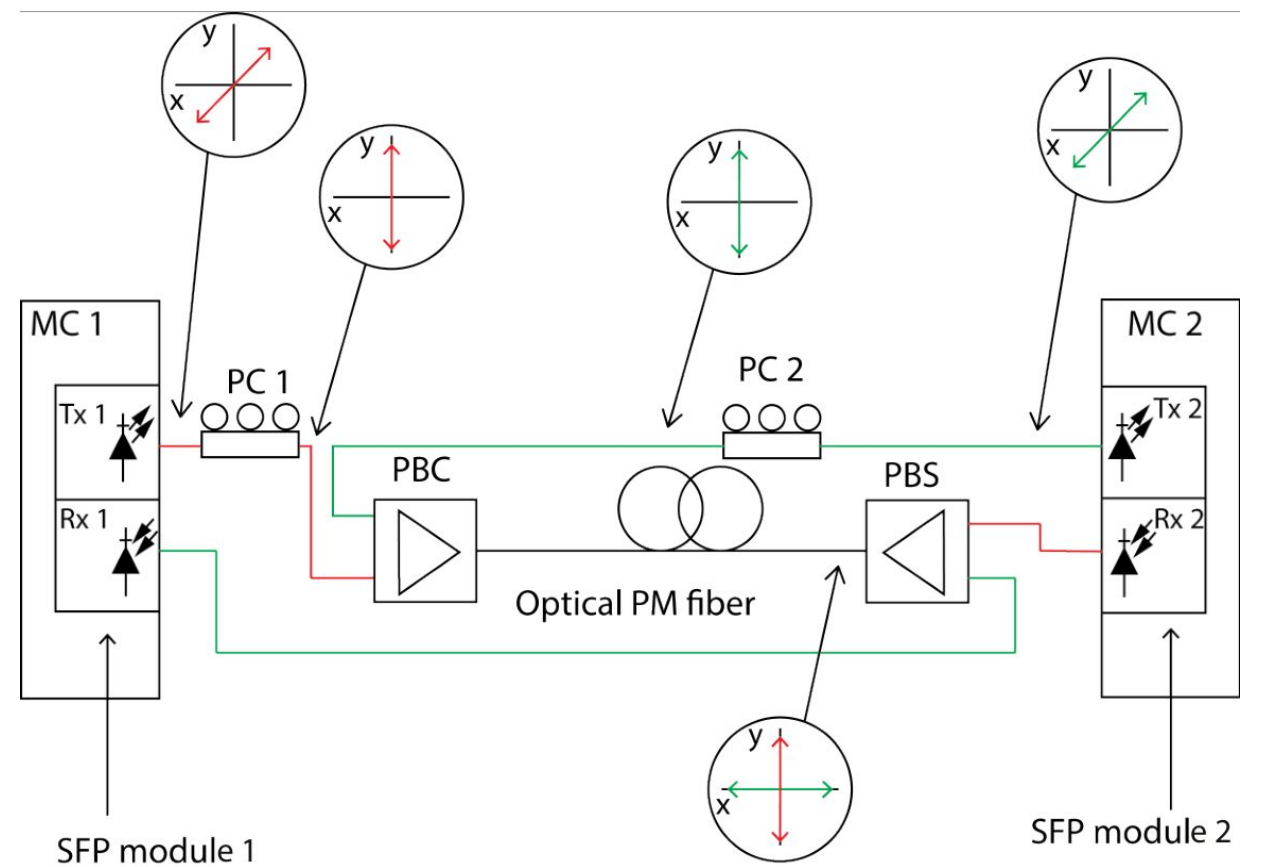

Fig. 2: Block diagram of the measured workplace, where MC1 and MC2 are media-convertors TP-LINK MC220L with 10032SM-LR80 SFP modules, PC1 and PC2 are realized by SM optical fiber controller, PBC and PBS are $1550 \mathrm{~nm} \mathrm{1x2}$ Polarization-Maintaining Fiber Optic Couplers by Thorlabs.

In case shown on the Fig. 2 (polarization maintaining fiber trace) there are no problems with synchronization of both media-convertors. The system operates well without collisions. Thanks to polarimeter we can control the polarization states in each connect point of our measurement trace. The theoretical assumption goes really down well and the polarization multiplex is functional. The on-line states of polarization are shown on Fig. 3 a) and $3 \mathrm{~b}$ ). 
The following research will be directed to use conventional single-mode fiber (standard G652.d) as the main transmission route. We suppose the necessity of dynamic polarization controller to ensure the correct functionality of polarization demultiplexer.

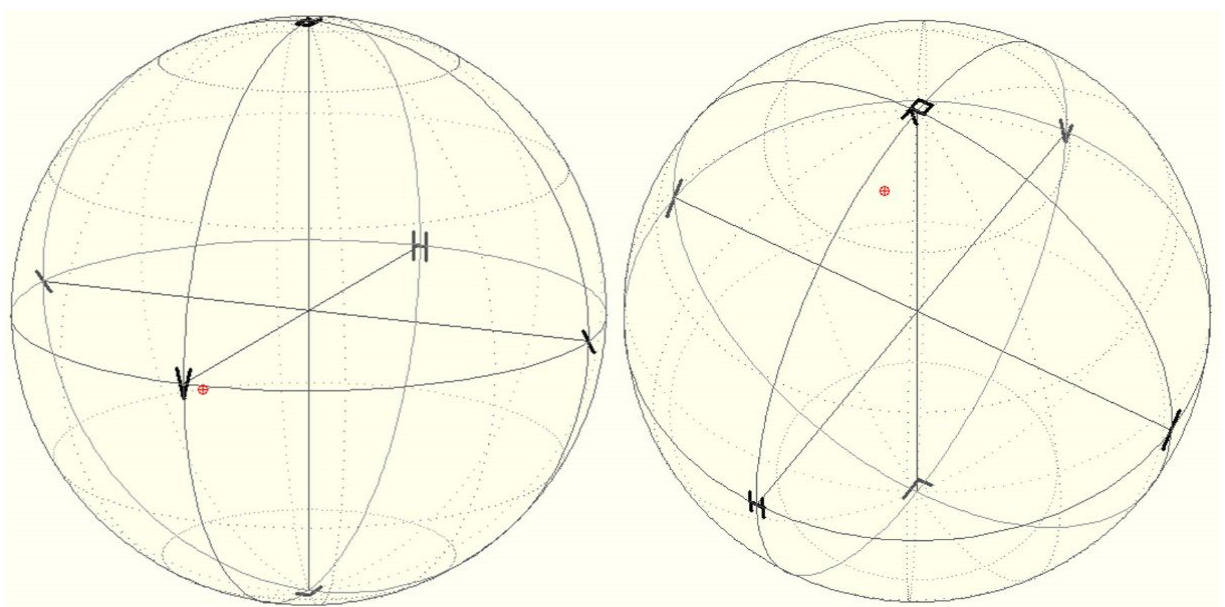

Fig 3: On-line states of polarization by polarimeter PAX5710IR3-T by Thorlabs:

a) Optical signal from Tx1 on input of PM coupler (vertically polarized light),

b) Multiplexed signal on output of PM coupler (circularly polarized light).

\section{CONCLUSION}

The text describes the theory of polarization multiplexing. It also included a proposal of possible arrangements of the experimental facility for the use of polarization planes as separate transmission channels. When using a polarization preserving fiber, the system was fully operational. Two independent signals are multiplexed by a polarizing splitter in polarization maintaining fibers. After passing through the second polarizing fiber polarization splitter he had served as a splitter and was able to split the signal.

The transmission speed using gigabit network elements is around 900 Mbit.s-1. When connecting single-mode fiber $5 \mathrm{~m}$ system did not work. Again first polarization splitter multiplex two independent signals, but the second polarizing splitter was not able to signal the multiplex despite efforts to correct the rotation signal when entering the second polarization splitters.

\section{ACKNOWLEDGMENT}

This work has been supported by Projects for the development of K217 and K207 Departments, Brno University of Defense - Modern electrical elements and systems and research of sensor and control systems to achieve battlefield information superiority.

\section{REFERENCES}

[1] Collet, E., [Field Guide to Polarization], SPIE Vol. FG05, 2005, ISBN ISBN 9780819458681

[2] Kumar, A. and Ghatak, A., [Polarization of Light with Applications in Optical Fibers], Wash.: SPIE, 227p. Tutorial texts in optical engineering, v. TT 90. ISBN 0819482153.

[3] Collet, E., [Polarized Light in Fiber Optics], SPIE, New Jersey 2003, ISBN 9780819457615.

[4] Filka, M., [Optoelectronics for Telecommunication and Informatics], Dallas, Texas, USA, 2009, ISBN 978-0615-33185-0 